\title{
Tumor regression grading after preoperative hyperfractionated radiotherapy/ chemoradiotherapy for locally advanced rectal cancers: interim analysis of phase III clinical study
}

\author{
Adam IDASIAK ${ }^{1, *}$, Katarzyna GALWAS-KLIBER ${ }^{1}$, Marcin RAJCZYKOWSKI $^{1}$, Iwona DĘBOSZ-SUWIŃSKA ${ }^{2}$, Marcin ZEMAN ${ }^{3}$, Ewa STOBIECKA ${ }^{4}$, \\ Jolanta MROCHEM-KWARCIAK ${ }^{5}$, Rafał SUWIŃSKI ${ }^{1}$ \\ ${ }^{1}$ II Clinic of Radiotherapy and Chemotherapy, Maria Sklodowska-Curie National Research Institute of Oncology, Gliwice Branch, Poland; \\ ${ }^{2}$ Radiotherapy Department, Maria Sklodowska-Curie National Research Institute of Oncology, Gliwice Branch, Poland; ${ }^{3}$ The Oncologic and \\ Reconstructive Surgery Clinic, Maria Sklodowska-Curie National Research Institute of Oncology, Gliwice Branch, Poland; ${ }^{4}$ Tumor Pathology \\ Department, Maria Sklodowska-Curie National Research Institute of Oncology, Gliwice Branch, Poland; ${ }^{5}$ Analytics and Clinical Biochemistry \\ Department, Maria Sklodowska-Curie National Research Institute of Oncology, Gliwice Branch, Poland
}

${ }^{*}$ Correspondence: adam.idasiak@io.gliwice.pl

Received December 17, 2020 / Accepted January 27, 2021

\begin{abstract}
We investigated the tumor regression grading (TRG) as a prognostic marker for disease-free survival (DFS) in patients with advanced rectal cancer treated within phase III randomized study (ClinicalTrials.gov Identifier: NCT01814969). The study is still recruiting prospective trial of preoperative hyperfractionated radiotherapy (HART) compared with concomitant hyperfractionated radiotherapy with co-administration of chemotherapy based on 5-FU (HART-CT) in patients with $\mathrm{T} 2 / \mathrm{N}+$ or T3/any $\mathrm{N}$ resectable rectal cancer. This preplanned interim analysis examined the pathological outcome in the group of 136 patients who were randomly assigned to HART $(n=69)$ and HART-CT $(n=67)$. The pelvis was irradiated twice a day (28 fractions of $1.5 \mathrm{~Gy}$ ), with a minimal interfraction interval of $8 \mathrm{~h}$ to a total dose of 42 Gy over 18 days (HART) or mentioned scheme with concurrent chemotherapy: 5-FU $325 \mathrm{mg} / \mathrm{m} 2$ (bolus) on days 1-3 and days 16-18 (HART-CT). Surgery was performed 6-7 weeks after HART/HART-CT. Postoperative 5-FU-based chemotherapy was given to ypN positive patients. The TRG was recorded using the following 4-point scale: TRG0 (pCR) denoted no cancer cells; TRG1 was diagnosed when a few cancer foci had been seen in less than $10 \%$ of a tumor mass; TRG2 denoted cancer cells seen in $10-50 \%$ of a tumor mass; in order to diagnose TRG3, cancer cells had to be seen in more than $50 \%$ of a tumor mass. Multivariable analysis was performed using Cox regression models and Cox proportional hazard model was used in the survival analysis. The crude rate of patients with any serious acute 3 toxicity during the follow-up was $16 \%$ vs. 25\% for HART and HART-CT. Twenty-two patients (16\%) presented with postoperative complications. Anterior resection was performed in $52 \%$ vs. $62 \%$ for HART and HART-CT respectively $(\mathrm{p}=0.06)$. Of the 136 patients evaluable for pathologic response, there were $3(4 \%)$ vs. $9(13 \%), 16(23 \%)$ vs. $24(36 \%), 40(58 \%)$ vs. $30(45 \%)$, and $10(15 \%)$ vs. $4(6 \%)$ patients with TRG 0, 1, 2, and 3 , respectively in HART vs. HART-CT, the difference was statistically significant $p=0.002$. The addition of 5 -FU infusion to HART was not associated with statistically significant improved loco-regional relapse-free survival (LRC), metastasis-free survival (MFS), and DFS. Significant differences in the tumor regression grading (TRG) were found. Both LRC and DFS of rectal cancer patients treated with HART vs. HART-CT had favorable outcomes in the HART-CT arm. Also, the sphincter preservation rate tended to favor HART-CT.
\end{abstract}

Key words: rectal cancer, hyperfractionated radiotherapy, histopathological response

The American Joint Commission on Cancer's (AJCC) pathologic tumor, node, metastasis (TNM) staging system is a common prognostic predictor used in patients undergoing preoperative radiotherapy (RT) or chemoradiotherapy (CHTRT). However, the TNM classification was not intended to provide information regarding the degree of tumor response to the preoperative RT/CHTRT. These
RT/CHTRT responses show variable results, ranging from complete pathological response to cancer progression and complete or near-complete pathological response, which predicts oncological outcomes [1-3].

Typically, downsizing and downstaging have been used to assess tumor response to RT or CHTRT, as indicated by a decrease in primary tumor size or the pathologic vs. 
pre-operative clinical $\mathrm{T}$ and $\mathrm{N}$ stage [4]. A conventional, long-course RT plan (about 50 Gy over 5 weeks) associated with chemotherapy (CHTRT) causes tumor downstaging when surgery is delayed due to the severe treatment reaction. Contrary to the assumption that hypofractionated short-course RT (25 Gy in 1 week) did not downstage tumor. Nevertheless, in some retrospective $[5,6]$ and prospective [7] studies, if the surgery is delayed for more than 4-5 weeks, short-course RT causes the downstaging. Tumor Regression Grading (TRG) is also observed in the combination of after preoperative hyperfractionated radiotherapy (HART) with an intravenous bolus of 5-FU, as we have previously shown [8]. That factor has been able to classify HARTCT tumor response and predict prognosis at the individual patient level.

The present work is a preplanned interim analysis in which we examine the pathological outcomes in the group of 136 patients in a randomized phase III trial (ClinicalTrials.gov Identifier: NCT01814969). We are going to further evaluate the safety and efficacy of accelerated hyperfractionated radiotherapy (HART) with concurrent 5-FU-based chemotherapy in patients with locally advanced rectal cancer. The present study focuses on primary outcome measures of the trial such as pathological complete response rate according to the TRG scale. The secondary endpoints included cumulative loco-regional relapse-free survival (LRC), as well as other secondary outcome measures, will be addressed in future reports.

\section{Patients and methods}

Study design and participants. The study (NCT01814969) was prospectively designed as a single institution two-arm, randomized phase III trial of preoperative concomitant accelerated hyperfractionated radiotherapy (HART) or HART with co-administration of two cycles of chemotherapy based on $5-\mathrm{FU}$ in patients with $\mathrm{T} 2$; $\mathrm{N} 1-\mathrm{N} 2$ or T3/ any $\mathrm{N}$ resectable mid-low primary rectal cancer. The study was approved by the local bioethical committee in June 2012 . All patients had given informed consent before recruitment. All patients had histologically confirmed mid-low rectal adenocarcinoma (within $12 \mathrm{~cm}$ from the anal verge), with no evidence of distant metastasis. Only the patients with resectable $\mathrm{T} 2 / \mathrm{N}+$ or $\mathrm{T} 3 /$ any $\mathrm{N}$ tumors were enrolled. Resectability and staging were assessed by a multidisciplinary team based on the clinical examination and CT/MRI imaging. The age at the diagnosis was between $18-75$ years. Patients were also required to have a World Health Organization (WHO) performance status of 0 or 1 with adequate liver, kidney, and bone marrow functions. Patients who had a prior history of chemotherapy or pelvic radiation therapy were excluded. Patients with a history of other malignancy within 5 years were also excluded. Other exclusion criteria included acute obstructive symptoms, unresectable disease, or any serious comorbidities deemed not suitable for chemoradiotherapy.
Patients underwent CT-simulation with $5 \mathrm{~mm}$ slices with a full bladder. The scan extended from the L4 vertebral body to below the perineum. A thermoplastic pelvic immobilization in a prone position was used to minimize the setup variability. Daily patients positioning was performed using skin markers and KV/DDR portal verification before each fraction. The gross target volumes (GTV) and clinical target volumes (CTV) were contoured on axial CT scan slices. GTV was defined as a primary tumor and involved lymph nodes. The CTV was defined as primary tumor, mesorectal region, presacral region, mesorectal lymph nodes, internal iliac lymph nodes. The external iliac lymph nodes were considered part of the CTV when there was major tumor extension into the internal and external anal sphincter. The radiation dose was prescribed to planning target volume (PTV). The PTV of the GTV and CTV were created by adding a $5 \mathrm{~mm}$ margin.

The total dose to be delivered to PTV was 42 Gy in 28 fractions of $1.5 \mathrm{~Gy}$, two times daily with at least $8 \mathrm{~h}$ interval. A three-field (with individualizing shields), IMRT (sliding window), or Rapid-Arc technique was used, all fields were treated during each fraction. High-energy photon beams of $20 \mathrm{MV}$ were used. Two cycles of chemotherapy were given concurrently with radiation therapy according to the scheme: $5-\mathrm{FU} 325 \mathrm{mg} / \mathrm{m}^{2}$ (bolus) on days $1-3$ and 16-18 (last 3 days of radiotherapy). In concern of possible overtreatment, the original protocol was, however, amended before the first recruitments, such that for $\mathrm{cN} 0$ patients only one cycle of 5-FU on days 1-3 was prescribed. Also, it was allowed to withhold 5-FU on days 16-18 for patients with grade III or IV acute side effects, if observed at the first course.

Surgery was performed 6-7 weeks after the completion of radiotherapy/chemoradiotherapy. The choice between abdominoperineal resection and anterior resection was left to the judgment of the surgeon as the function of primary tumor location and response to chemoradiotherapy was assessed before and during surgery. For node-positive patients' adjuvant 5-FU based chemotherapy was recommended after surgery to a total of 6 cycles.

Between January 2014 and June 2017, a total of 136 eligible patients were randomly assigned to preoperative HART $(n=69)$ and HART-CT $(n=67)$ accordingly with the protocol study. Patient and tumor characteristics are presented in Table 1. The groups were well balanced with respect to potential prognostic factors. Tumors were located within $10 \mathrm{~cm}$ from the anal verge in 129 patients (95\%). The median time interval between the end of the preoperative treatment and surgery was 6.7 weeks in both groups. The mean follow-up time was 2.2 years, median 2.0 years. Liver metastases were detected in 3 and 4 patients in the HART and HART-CT groups, respectively. The tumor was resected in 133 patients (97\%). An anterior resection was performed in 34 patients of $(49 \%)$ in the HART group and 38 patients $(57 \%)$ in the HART-CT group. 
Table 1. Characteristics of the patients.

\begin{tabular}{|c|c|c|c|c|c|c|}
\hline Parameter & & $\begin{array}{c}\text { HART } \\
(\mathrm{n}=69)(\%)\end{array}$ & $\begin{array}{l}\text { HART-CT } \\
(\mathrm{n}=67)(\%)\end{array}$ & $\begin{array}{c}\text { 2-yrs DFS } \\
(\%)\end{array}$ & $\mathbf{R R}$ & p-value $<0.05^{\star}$ \\
\hline \multirow[t]{2}{*}{ Gender } & Male & 39 & 38 & 70 & 1.65 & 0.19 \\
\hline & Female & 30 & 29 & 81 & & \\
\hline Age [median (range)] & & $65(36-77)$ & $62(38-80)$ & & & \\
\hline \multirow[t]{2}{*}{ c Tumor Stage } & c T2 & 2 & 0 & - & 1.08 & 0.57 \\
\hline & c T3 & 67 & 67 & 75 & & \\
\hline \multirow[t]{3}{*}{ c Nodal Stage } & c N0 & 16 & 12 & 69 & 1.59 & 0.90 \\
\hline & $\mathrm{cN1}$ & 28 & 34 & 85 & & \\
\hline & $\mathrm{c}$ N2 & 25 & 21 & 65 & & \\
\hline Number of cycles of 5-FU & 0 & 69 & 0 & 69 & 1.25 & 0.34 \\
\hline \multirow[t]{2}{*}{ during RT } & 1 & - & 19 & 85 & & \\
\hline & 2 & - & 48 & 78 & & \\
\hline \multirow{5}{*}{ yp Tumor stage } & урT0 & $2(3 \%)$ & $9(13 \%)$ & 100 & 4.51 & $0.002^{\star}$ \\
\hline & урT1 & $4(6 \%)$ & $4(6 \%)$ & 95 & & \\
\hline & урT2 & $22(32 \%)$ & $23(34 \%)$ & 91 & & \\
\hline & урT3 & $39(56 \%)$ & $29(43 \%)$ & 60 & & \\
\hline & урT4 & $2(3 \%)$ & $2(3 \%)$ & 50 & & \\
\hline \multirow[t]{3}{*}{ yp Nodal stage } & yp No & $37(54 \%)$ & $42(63 \%)$ & 84 & 2.95 & $0.015^{\star}$ \\
\hline & yp N1 & $20(29 \%)$ & $19(28 \%)$ & 64 & & \\
\hline & yp N2 & $12(32 \%)$ & $6(9 \%)$ & 62 & & \\
\hline \multirow[t]{4}{*}{ TRG scale } & 0 & $3(4 \%)$ & $9(13 \%)$ & 91 & 2.58 & $0.006^{*}$ \\
\hline & 1 & $16(23 \%)$ & $24(36 \%)$ & 95 & & \\
\hline & 2 & $40(58 \%)$ & $30(45 \%)$ & 64 & & \\
\hline & 3 & $10(15 \%)$ & $4(6 \%)$ & 50 & & \\
\hline \multirow[t]{2}{*}{ Postoperative chemotherapy } & No & $32(46 \%)$ & $35(53 \%)$ & 90 & 3.45 & $0.002^{*}$ \\
\hline & Yes & $37(54 \%)$ & $32(47 \%)$ & 58 & & \\
\hline \multirow[t]{3}{*}{ CEA before treatment } & $\leq 5 \mathrm{ng} / \mathrm{ml}$ & $44(64 \%)$ & $42(63 \%)$ & 79 & 3.63 & 0.06 \\
\hline & $>5 \mathrm{ng} / \mathrm{ml}$ & $22(32 \%)$ & $23(34 \%)$ & 66 & & \\
\hline & missing & $3(4 \%)$ & $2(3 \%)$ & - & & \\
\hline \multirow[t]{3}{*}{ Type of surgery } & Anterior resection & $36(52 \%)$ & $42(62 \%)$ & 86 & 3.48 & $0.006^{*}$ \\
\hline & Abdominoperineal resection & $31(45 \%)$ & $22(34 \%)$ & 66 & & \\
\hline & Other & $2(3 \%)$ & $3(4 \%)$ & - & & \\
\hline
\end{tabular}

Abbreviations: HART - hyperfractionated radiotherapy; HART-CT - concomitant hyperfractionated radiotherapy with co-administration of two cycles of chemotherapy based on 5-FU; CEA - carcinoembryonic antigen; RR - relative risk

Pathologic examination and definition of TRG. For the evaluation of the HART/HART-CT effect in residual cancer, the tumor regression grading (TRG) was used. The TRG scale is based on the microscopic evaluation of the presence of residual tumor cells in relation to the extension of fibrosis using the following 4-point scale as recommended by the AJCC Cancer Staging Manual, $7^{\text {th }}$ Edition as follows: TRG 0 (pCR) denoted no cancer cells; TRG 1 was diagnosed when a few cancer foci had been seen in $<10 \%$ of a tumor mass; TRG 2 denoted cancer cells seen in $10-50 \%$ of tumor mass; in order to diagnose TRG 3, cancer cells had to be seen in $>50 \%$ of tumor mass. The pathology evaluation included assessment of ypT category, ypN category, grade of tumor malignancy, and status of resection margins and TRG scale.

Statistical analysis. An interim analysis was designed when the first group of 130 patients had completed all therapies and was followed up at least 12 months. The associations between 2-year DFS and pretreatment clinicopathological characteristics, sphincter preservation rate, and postsurgical pathologic factors were assessed, according to the treatment arm.

DFS was defined as the time between randomization and either loco-regional recurrence, distant metastases, progression, or death from any cause, whichever occurred first. Locoregional failure was defined as the recurrence of cancer in the irradiated area. Local recurrence was defined as the relapse within the original location of the tumor, nodal recurrence of cancer was defined as the failure within the lymphatic system of the pelvis. Distant metastases were defined as a relapse outside the irradiated area. OS was calculated from the date of study entry to the date of death from any cause or to the date of last follow-up. The cumulative incidence of loco-regional and distant recurrence was defined as the time between random assignment and occurrence of any loco- 
Table 2. Tumor regression grading in patients treated with preoperative HART/HART-CT.

\begin{tabular}{llccc}
\hline Parameter & & $\begin{array}{c}\text { HART } \\
(\mathbf{n = 6 9 )}(\%)\end{array}$ & $\begin{array}{c}\text { HART-CT } \\
(\mathbf{n = 6 7 )}(\%)\end{array}$ & p-value \\
\hline TRG scale & 0 & $3(4 \%)$ & $9(13 \%)$ & 0.002 \\
$(4$-level TRG) & 1 & $16(23 \%)$ & $24(36 \%)$ & \\
& 2 & $40(58 \%)$ & $30(45 \%)$ & \\
& 3 & $10(15 \%)$ & $4(6 \%)$ & 0.001 \\
TRG scale & $0+1$ & $19(28 \%)$ & $33(49 \%)$ & \\
$(2-$ level TRG) & & & & \\
& $2+3$ & $50(72 \%)$ & $34(51 \%)$ & \\
\hline
\end{tabular}

Table 3. Multivariate analyses of the factors for 2-year DFS (disease-free survival).

\begin{tabular}{lcc}
\hline Factors & Hazard ratio $(\mathbf{C I})$ & $\begin{array}{c}\text { p-value } \\
<\mathbf{0 . 0 5}\end{array}$ \\
\hline yp Tumor stage & $1.138(0.610-2.120)$ & 0.0683 \\
yp Nodal stage & $0.996(0.551-1.801)$ & 0.990 \\
Operation method (type of surgery) & $1.703(1.008-2.877)$ & $\mathbf{0 . 0 4 6}^{*}$ \\
Postoperative chemotherapy & $2.743(1.413-5.328)$ & $\mathbf{0 . 0 0 2}^{\star}$ \\
Tumor Regression Grade & $2.536(1.241-5.181)$ & $\mathbf{0 . 0 1 0}^{\star}$ \\
\hline
\end{tabular}

regional recurrence and distant recurrence, respectively, irrespective of whether this was the first event or not. We performed univariate analyses using the log-rank test, stratified by treatment arm. The survival curves have been plotted using the Kaplan-Meier method and were compared using the Cox test. The log-rank test was used to test the significance level, with a value of $\mathrm{p}<0.05$ considered to be statistically significant.

\section{Results}

Pathologic response (TRG). In the group of 136 patients evaluable for pathologic response there were $3(4 \%)$ vs. 9 (13\%); $16(23 \%)$ vs. 24 (36\%); 40 (58\%) vs. $30(45 \%)$; and 10 (15\%) vs. 4 (6\%) patients with TRG $0,1,2$, and 3 , respectively in HART vs. HART-CT, the difference was statistically significant ( $\mathrm{p}=0.002$ ). Furthermore, when TRG was combined into 2 two groups (TRG $0-1$ or TRG $2-3$ ) there was still a statistically significant difference in the incidence found in both groups ( $\mathrm{p}=0.001$ ) The appropriate results of TRG after both treatments are shown in Table 2.

The results of TRG for two-level grouped TRG (TRG 0+1 vs. TRG 2+3), HART-CT led to a statistically significant shift toward lower TRG groups compared with the HART arm, ( $49 \%$ vs. $27 \%$ ), respectively.

Acute toxicity. The medical history of 136 patients was available for toxicity evaluation. Severe acute Grade 3 toxicity including diarrhea was found in 6 patients $(9 \%)$ in the HART arm and $8(12 \%)$ in the HART-CT arm $(\mathrm{p}=0.11)$. Grade $1-2$ proctitis was reported in $30(43 \%)$ and Grade 3 in 3 patients (4\%) in the HART arm vs. 36 (54\%) with Grade 1-2 proctitis, and 5 patients (7\%) with Grade 3 proctitis in the HART-CT arm. Proctitis did not require chemoradiotherapy interruption and was controlled with nonsteroidal anti-inflammatory drugs.

The hematologic toxicity Grade 3 including neutropenia was found in 2 patients (3\%) in the HART arm and 4 patients (6\%) in the HART-CT arm ( $\mathrm{p}=0.005)$. There was no Grade 4 toxicity reported as well no renal, liver, and alopecia were observed in both arms.

Surgical procedures by the treatment arm. Surgery was performed at the median interval of 49 days (range 24-94). Although the recommended interval surgery-radiotherapy was 6-7 weeks, 16 patients (12\%) underwent surgery earlier or later mainly owing patients or surgeon preference. Sphincter-saving surgery was performed in 78 patients (57\%). Anterior resection was performed in $52 \%$ vs. $62 \%$ for HART and HART-CT, respectively. No significant differences were found in the type of surgery by the treatment arm $(p=0.06)$. All primary tumor resections had negative proximal, distal, and circumferential resection margins (R0 resections).

No particular complications were encountered intraoperatively. Twenty-two patients (16\%) presented with postoperative complications (during 3 months after surgery). Four patients with postoperative wound infections, four cases of anastomotic fistulae (required surgical interventions), four cases of delayed healing of the wounds, three recto-vaginal and one recto-perineal fistulas managed surgically, three developed a rectal abscess, two cases of urinary infection, and one case of small bowel obstruction (treated conservatively). There was no postoperative death in the analyzed group of patients. There were no significant differences in the postoperative complications rate by the treatment arm.

Adjuvant chemotherapy. Sixty-nine patients of the analyzed group (51\%) received adjuvant chemotherapy. There were no significant differences in the rate treated with adjuvant 5-FU based postoperative chemotherapy by the treatment arm.

Other endpoints-DFS, LRC, MFS. All 136 patients were included in the interim survival analysis on an intent-totreat basis. We investigated the prognostic significance of the treatment arms and various clinicopathologic factors affecting DFS in univariate analysis (Table 1).

The addition of 5-FU infusion to HART was not associated with statistically significant improved loco-regional relapse-free survival (LRC), metastasis-free survival (MFS), and disease-free survival (DFS). These data will be subjects for further analysis after the end of recruitment to the study.

When TRG was combined into 2 two groups (TRG $0-1$ or TRG 2-3) there was a significant difference in outcomes constituted an independent prognostic factor for DFS. This included 2-year DFS: TRG 0-1: 94\% vs. TRG 2-3: 60\%; $\mathrm{p}=0.0006$ (Figure 1). Significant differences in 2-year MFS: TRG 0-1: 94\% vs. TRG 2-3: 70\%; $\mathrm{p}=0.0027$ (Figure 2) and 2-year LRC: TRG 0-1: 96\% vs. TRG 2-3: $82 \%$; $\mathrm{p}=0.014$ were also found (Figure 3 ). 
The univariate analysis indicated that the factors associated with 2-year disease-free survival consisted of ypT category, ypN category, type of operation, postoperative chemotherapy, and TRG (Table 1). On multivariate analysis, operation method, postoperative chemotherapy, and TRG were independent prognostic factors for disease-free survival (Table 3).

\section{Discussion}

For a number of gastrointestinal tumors, preoperative treatment has become standard practice. Tumor response to preoperative therapy displays a wide spectrum of ranges, from histopathologically confirmed completed regression of tumor to lack of response. In rectal cancer, the response of the tumor depends on many factors, such as the dosage and schedule of preoperative care (chemotherapy, RT, or combination of them), the time between treatment and surgery, and tumor biology [9-11]. Tumor downstaging may matter for advanced disease resectability and may affect sphinctersaving surgery percentage. In the current series, the levels of complete pathological response are 13\% in the HART-CT and $4 \%$ in the HART arm, which is similar to that stated by Gerard [12].

According to the authors, the rate of completed responses is close to $0 \%$ after short course irradiation and immediate surgery. It is $<10 \%$ with preoperative radiotherapy alone and the long interval before surgery and reaches 15-19\% with recent chemo-radiotherapy protocols. Nonetheless, there are many concerns about the translation of enhanced pathological responses into increasing the rate of sphinctersaving surgery, disease-free, or overall survival. Several studies have shown a better prognosis in patients with significant tumor regression after RT/CHTRT, particularly in ones with pathological complete response $[13,14]$. Obviously, new studies on this topic may allow this issue to be better addressed. Lack of tumor regression means an aggressive malignant phenotype and therefore also a higher metastatic risk. The tendency to increase metastases by the biological mechanisms mediating local resistance to HART/HART-CT would be a significant consequence of this principle. The processes behind those clinical studies remain unclear. According to some authors, cancer cell death induced by radiation can activate the innate immune system, whereas increased cytotoxic-T cells infiltration has been documented in rectal cancer after RT/CHTRT [3, 15, 16]. For this reason, the correlation between TRG $0-1$ and the longer interval between completion of preoperative radio/radio-chemotherapy and surgery may indicate an activated immune response, which exerts its antitumorigenic effect continuously while awaiting surgery. This may also clarify the connection between lower TRG and lower metastasis incidence. Although the potentially significant prognostic value of TRG in preoperative CHTRT-treated rectal cancer has recently been suggested, TRG efficacy is

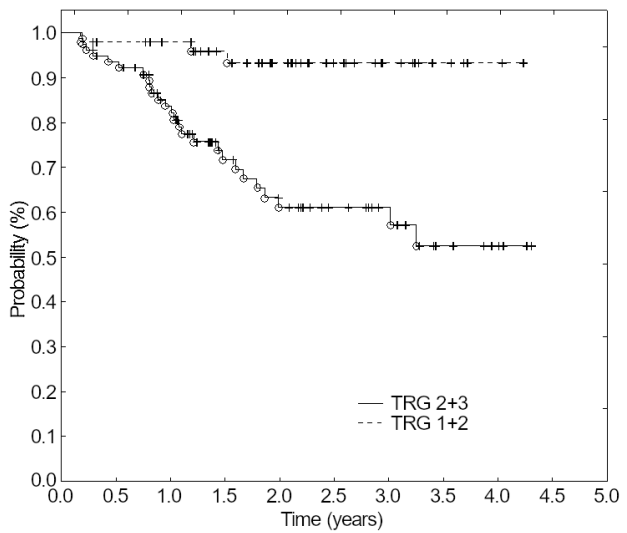

Figure1. 2-year disease-free survival: TRG 0-1: 94\% vs. TRG 2-3: 60\%; $p=0.0006$

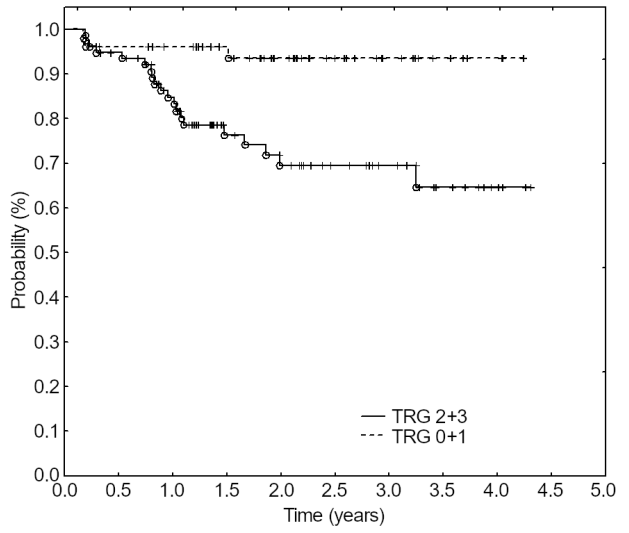

Figure 2. 2-year metastasis-free survival: TRG 0-1: 94\% vs. TRG 2-3: $70 \% ; \mathrm{p}=0.0027$

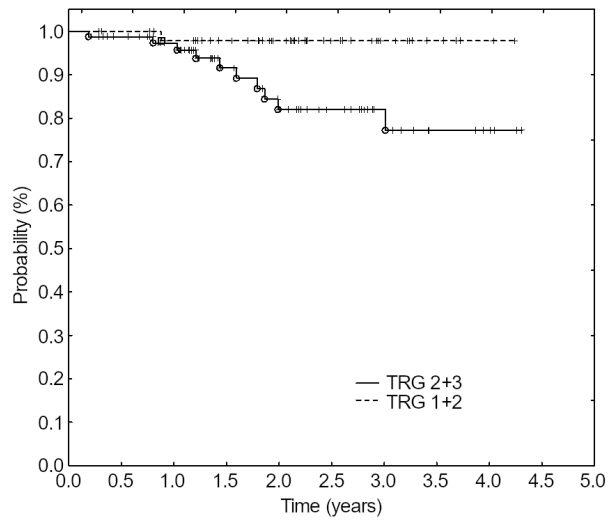

Figure 3. 2-year loco-regional control: TRG 0-1: 96\% vs. TRG 2-3: 82\%; $\mathrm{p}=\mathbf{0 . 0 1 4}$

still not well understood, widely debated, and remains a subject of controversy as an accurate and reliable prognostic factor. Four tumor-related, pathological factors including 
ypT, ypN, ypStage, and TRG in 420 rectal cancer patients treated with preoperative CHTRT were compared by Kim et al. The authors discovered that $\mathrm{ypN}$ was the most dominant indicator for disease-free survival prediction when TRG has the least prognostic ability [17]. Rodel et al. also confirmed that TRG alone is not a statistically important prognostic factor [18]. Our findings, however, support the results of many previous studies, which showed TRG as an important prognosis indicator with preoperative CHTRT treatment for patients with rectal cancer. This interim analysis shows that using AJCC criteria, TRG is an independent prognostic factor for 2-year disease-free survival on analysis of 136 patients with a documented history of rectal cancer treated with preoperative HART/HART-CT. The conclusions of this study indicate that hyperfractionated chemo-radiotherapy (42 Gy provided over 18 days in $1.5 \mathrm{~Gy}$ fractions at the same time as 5-FU dependent chemotherapy) is workable and gives a satisfactory short-term result. With a $100 \%$ radiotherapy compliance rate and occasional diarrhea that did not need a treatment break, the acute toxicity is acceptable. The toxicity profile associated with standard RT combined with chemotherapy is similar to these data [19-22]. From our experience, the suggested schedule for standard radiochemotherapy (25-30 fractions once daily) can reduce radiotherapy time from 5-6 weeks to 14 working days. On the other hand, the prescription of 5-FU bolus given simultaneously with radiotherapy may be considered as similarly efficient to continuous infusion [23]. From this point of view, the proposed therapy may be particularly beneficial for patients living far away from hospital facilities, due to the short stay in the hotel. Probably even more comfortable is very short hypofractionated radiotherapy alone, but it comes at the expense of potential rises in late toxicity and the lack of systematic therapy. In view of the fact that only subgroups of patients can take advantage of such forms of treatment, it is clear that concurrent chemotherapy and HART require further analysis before any broader introduction.

To sum up, TRG 0-1 following preoperative HART/ HART-CT was correlated with statistically better patient outcomes and delivered more details after adjusting for the treatment arm, pathological stage, and local resection status. TRG with its early availability, which is particularly useful in times of personalized medicine, can help to validate predictive biomarkers, recognize the most valuable pre-operative treatment strategy for large-scale testing in early clinical trials and simplify response-guided therapeutic strategies. The TRG based on AJCC criteria is an independent and convincing prognostic factor for disease-free survival in patients with rectal cancer treated with HART/HART-CT preoperatively. TRG can also be a useful prognostic predictor for individualizing the prognosis and potentially guiding postoperative treatment of resected rectal cancer patients who have undergone preoperative radiotherapy/ chemoradiotherapy.

\section{References}

[1] VECCHIO FM, VALENTINI V, MINSKY BD, PADULA GD, VENKATRAMAN ES et al. The relationship of pathologic tumor regression grade (TRG) and out comes after preoperative therapy in rectal cancer. Int J Radiat Oncol Biol Phys 2005; 62: 752-760. https://doi.org/10.1016/j. ijrobp.2004.11.017

[2] HUEBNER M, WOLFF BG, SMYRK TC, AAKRE J, LARSON DW. Partial pathologic response and nodal status as most significant prognostic factors for advanced rectal cancer treated with preoperative chemoradiotherapy. World J Surg 2012; 36: 675-683. https://doi.org/10.1007/s00268-0111409-8

[3] FOKAS E, STROBEL P, FIETKAU R, GHADIMI M, LIERSH $\mathrm{T}$ et al. Tumor regression grading after preoperative chemoradiotherapy as a prognostic factor and individual-level surrogate for disease-free survival in rectal cancer. J Natl Cancer Inst 2017: 109. https://doi.org/10.1093/jnci/djx095

[4] YEO SG, KIM DY, PARK JW, OH JH, KIM SY et al. Tumor volume reduction rate after preoperative chemoradiotherapy as a prognostic factor in locally advanced rectal cancer. Int J Radiat Oncol Biol Phys 2012; 82: e193-199. https://doi. org/10.1016/j.ijrobp.2011.03.022

[5] PETTERSSON D, HOLM T, IVERSEN H, BLOMQVIST L, GLIMELIUS B et al. Preoperative short-course radiotherapy with delayed surgery in primary rectal cancer. Br J Surg 2012; 99: 577-583. https://doi.org/10.1002/bjs.7796

[6] RADU C, BERGLUND A, PAHLMAN L, GLIMELIUS B. Short-course preoperative radiotherapy with delayed surgery in rectal cancer - a retrospective study. Radiother Oncol 2008; 87: 343-349. https://doi.org/10.1016/j.radonc.2007.11.025

[7] PACH R, KULIG J, RICHTER P, GACH T, SZURA M et al. Randomized clinical trial on preoperative radiotherapy $25 \mathrm{~Gy}$ in rectal cancer - treatment results at 5-year followup. Langenbecks Arch Surg 2012; 397: 801-807. https://doi. org/10.1007/s00423-011-0890-8

[8] IDASIAK A, GALWAS-KLIBER K, BEHRENDT K, WZIETEK I, KRYJ M et al. Pre-operative hyperfractionated concurrent radiochemotherapy for locally advanced rectal cancers: a phase II clinical study. Br J Radiol 2017; 90: 20160731. https://doi.org/10.1259/bjr.20160731

[9] CHETTY R, GILL P, BATEMAN AC, DRIMAN DK, GOVENDER D et al. Pathological grading of regression: An International Study Group perspective. J Clin Pathol 2012; 65: 865-866. https://doi.org/10.1136/jclinpath-2012-201054

[10] PETTERSSON D, LORINC E, HOLM T, IVERSEN H, CEDERMARK B et al. Tumour regression in the randomized Stockholm III Trial of radiotherapy regimens for rectal cancer. Br J Surg 2015; 102: 972-978; discussion 978. https://doi. org/10.1002/bjs.9811

[11] MACE AG, PAI RK, STOCCHI L, KALADY MF. American Joint Committee on Cancer and College of American Pathologists regression grade: A new prognostic factor in rectal cancer. Dis Colon Rectum 2015; 58: 32-44. https://doi. org/10.1097/DCR.0000000000000266 
[12] GERRARD JP, ROSTOM Y, GAL J, BENCHIMOL D, ORTHOLAN C et al. Can we increase the chance of sphincter saving surgery in rectal cancer with neoadjuvant treatments: lessons from a systematic review of recent randomized trials. Crit Rev Oncol Hematol 2012; 81: 21-28. https://doi. org/10.1016/j.critrevonc.2011.02.001

[13] RODEL C, MARTUS P, PAPADOUPOLOS T, FUZESI L, KLIMPFINGER $M$ et al. Prognostic significance of tumor regression after preoperative chemoradiotherapy for rectal cancer. J Clin Oncol 2005; 23: 8688-8696. https://doi. org/10.1200/JCO.2005.02.1329

[14] MAAS M, NELEMANS PJ, VALENTINI V, DAS P, RODEL $C$ et al. Long-term outcome in patients with a pathological complete response after chemoradiation for rectal cancer: a pooled analysis of individual patient data. Lancet Oncol 2010; 11: 835-844. https://doi.org/10.1016/S14702045(10)70172-8

[15] TENG F, MU D, MENG X, KONG L, ZHU H et al. Tumor infiltrating lymphocytes (TILs) before and after neoadjuvant chemoradiotherapy and its clinical utility for rectal cancer. Am J Cancer Res 2015; 5: 2064-2074.

[16] SHARABI AB, LIM M, DEWEESE TL, DRAKE CG Radiation and checkpoint blockade immunotherapy: Radiosensitisation and potential mechanisms of synergy. Lancet Oncol 2015; 16: e498-509. https://doi.org/10.1016/S14702045(15)00007-8

[17] KIM TH, CHANG HJ, KIM DY, JUNG KH, HONG YS et al. Pathologic nodal classification is the most discriminating prognostic factor for disease-free survival in rectal cancer patients treated with preoperative chemoradiotherapy and curative resection. Int J Radiat Oncol Biol Phys 2010; 77: 1158-1165. https://doi.org/10.1016/j.ijrobp.2009.06.019
[18] RODEL C, MARTUS P, PAPADUOPOLOS T, FUZESI L, KLIMPFINGER $\mathrm{M}$ et al. Prognostic significance of tumor regression after preoperative chemoradiotherapy for rectal cancer. J Clin Oncol 2005; 23: 8688-8696. https://doi. org/10.1200/JCO.2005.02.1329

[19] SAUER R, BECKER H, HOHENBERGER W, RODEL C, WITTEKIND $\mathrm{C}$ et al. Preoperative versus postoperative chemoradiotherapy for rectal cancer. N Engl J Med 2004; 351: 1731-1740. https://doi.org/10.1056/NEJMoa040694

[20] BOSSET JF, CALAIS G, MINEUR L, MAINGON P, RADOSEVIC-JELIC L et al. Enhanced tumoricidal effect of chemotherapy with preoperative radiotherapy for rectal cancer: preliminary results-EORTC 22921. J Clin Oncol 2005; 23: 5620-5627. https://doi.org/10.1200/JCO.2005.02.113

[21] BOSSET JF, COLLETTE L, CALAIS G, MINEUR L, MAINGON P et al. Chemotherapy with preoperative radiotherapy in rectal cancer. N Engl J Med 2006; 35: 1114-1123. https:// doi.org/10.1056/NEJMoa060829

[22] GERARD JP, CONROY T, BONNETAIN F, BOUCHE O, CHAPET $\mathrm{O}$ et al. Preoperative radiotherapy with or without concurrent fluorouracil and leucovorin in T3-4 rectal cancers: results of FFCD 9203. J Clin Oncol 2006: 24: 46204625. https://doi.org/10.1200/JCO.2006.06.7629

[23] SMALLEY SR, BENEDETTI JK, WILLIAMSON SK, ROBERTSON JM, ESTES NC et al. Phase III trial of fluorouracil-based chemotherapy regimens plus radiotherapy in postoperative adjuvant rectal cancer: GI INT 0144. J Clin Oncol 2006; 24: 3542-3547. https://doi.org/10.1200/ JCO.2005.04.9544 